\title{
Un centre de ressources pour comprendre les entreprises du temps présent...
}

Entretiens avec G. MOURADIAN et A. DESPLANQUE, Centre des Archives du Monde du Travail

\section{(2) OpenEdition \\ Journals}

Édition électronique

URL : http://journals.openedition.org/edc/2504

DOI : 10.4000/edc. 2504

ISSN : 2101-0366

Éditeur

Université Lille-3

Édition imprimée

Date de publication : 1 janvier 1995

Pagination : 183-193

ISSN : 1270-6841

Référence électronique

"Un centre de ressources pour comprendre les entreprises du temps présent... », Études de

communication [En ligne], 16 | 1995, mis en ligne le 19 décembre 2011, consulté le 02 mai 2019. URL

http://journals.openedition.org/edc/2504; DOI : 10.4000/edc.2504

Ce document a été généré automatiquement le 2 mai 2019.

(c) Tous droits réservés 


\section{Un centre de ressources pour comprendre les entreprises $\mathrm{du}$ temps présent...}

Entretiens avec G. MOURADIAN et A. DESPLANQUE, Centre des Archives du Monde du Travail

\section{Entretien avec G. Mouradian, directeur des Archives du Monde du Travail}

$1 \quad$ Les chercheurs des sciences de la communication ne sont pas, pour la plupart, des historiens. Ils travaillent le plus souvent sur des phénomènes contemporains, cherchent à comprendre des évolutions, des mutations. Que ce soit à propos de vie locale, d'entreprises, de médias...

Pourtant, pour ne pas être prisonniers d'une illusion de "modernité», il leur faut souvent adopter le regard historique qui cherche les lignes de fracture, les répétitions, les situations où «plus ça change, plus c'est toujours la même chose»...

Aussi Etudes de Communication a voulu, dans cette perspective, ouvrir une nouvelle rubrique en faisant connaitre aux chercheurs un nouvel outil pour leurs travaux: les Archives du Monde $d u$ Travail, ouvert au public depuis une bonne année, à Roubaix.

2 P. D. : Le titre "Archives du Monde du Travail» est une nouvelle dénomination, en France. Quel projet institutionnel recouvre un tel terme?

3 Georges Mouradian: La notion française s'est constituée dans les années 1970, quand on s'est préoccupé vraiment de trouver d'autres sources que les sources d'Archives publiques - de façon importante en tous cas, puisque la collecte d'archives d'entreprises date de l'entre-deux guerres. Le mouvement de 68 a incité à ouvrir de nouveaux champs à la recherche et à trouver des sources issues directement des acteurs sociaux de l'entreprise : syndicats, associations, entreprises. S'y est ajouté la crise, la casse industrielle, qui ont abouti à la fermeture d'entreprises. On s'est alors préoccupé, dans l'urgence, de la mémoire des archives privées produites par l'entreprise. En France on a développé le concept du «Monde du travail», officialisé comme terminologie dans les années 80 . Cette 
notion est assez différente de celle qui est développée à l'étranger où il y a une césure assez forte entre les archives d'entreprise (Business Archives selon le terme anglo-saxon) et les Centres d'Histoi-re Sociale où associations, organismes du mouvement syndical et partis politiques se sont préoccupés de regrouper leurs documentations d'abord, leurs archives ensuite. En France, on a considéré que les Archives du Monde du Travail étaient celles des acteurs sociaux de la production, ceux qui interviennent dans le champ des rapports sociaux de production de manière organisée ou à titre personnel. On pourra donc ici trouver aussi des archives personnelles de militants, des archives personnelles patronales.

Ainsi, en France on a trouvé qu'il était plus pertinent de regrouper les deux versants du procès de production: archives d'entreprise -économiques -, archives syndicales.

t
trouvera en Belgique, aux Pays-Bas, en Allemagne, en Italie... où il y a une césure entre l'entreprise et le mouvement ouvrier au sens large, qu'il soit d'obédience chrétienne, social-démocrate ou marxiste. En France ce sont les archives nationales et départementales, mais aussi des institutions autour de l'Université, l'Institut Historique du Temps Présent notamment, qui collectent les archives politiques... et cherchent d'ailleurs à collecter aussi les archives syndicales, sur le mode de leurs collègues étrangers.

P. D. : Quelle est la nature des supports que vous collectez?

7 G. $M$ : Les archives, au sens de l'article premier de la loi de 79, n'excluent pas un support ou un autre, tant que les documents portent de l'information et sont produits dans le cadre d'une action. A priori on n'a pas vocation - mais, certes, il y a des problèmes frontaliers -, à être un centre de mémoire pour une ethnographie du monde de travail, par exemple. On trouvera essentiellement le support papier, la vidéo, la photo, mais encore parfois des documents qui se rapprochent de l'objet - d'où les problèmes frontaliers que j'évoquais - registres d'échantillons de filatures, logos, étiquettes, documents techniques. Il s'agit là de documents qui sont intimement liés à la production de l'entreprise et sont donc Archives de l'entreprise... et en même temps les Musées des Arts et des Techniques, les Musées d'Arts Appliqués s'intéresseront aussi à la collecte de ces documents. Autant alors ne pas se situer en termes de rivalité et faire comprendre ce que notre collecte a de spécifique : une institution muséographique, quand elle procède à la collecte de ce genre d'objet va avoir tendance à détruire la partie archives papier, écrites, pour ne collecter que cela. Alors que nous, autant s'accorder de temps en temps un peu de mérite, on a effectivement une action cohérente sur cet ensemble de documentation. Un document vaut dans son contexte de production.

Il faut ajouter un point : nous nous préoccupons de collecte pérenne. Les Archives du Monde du Travail ont été créées au cœur de la crise de la société industrielle, on pourrait dire dès lors que c'est une institution de travail de deuil, occupée à collecter de manière un peu nécrologique la mémoire d'un monde qui disparaît... Soit. Mais, en tout cas, notre but est bien de produire de nouvelles sources, des sources permanentes de notre époque. Je ne suis pas sûr qu'un Ecomusée - qui fait un travail de représentation de la société industrielle, travail de mémoire pour des gens qui sont encore en contact - soit sur la même problématique quand il contribue à la création d'un centre d'archives. Le plus souvent, il le fait à l'occasion d'une action de sauvetage, et le danger est de n'inscrire 
cette collecte que dans une action immédiate ou de courte durée, avec le danger de morcellement que j'indiquais tout à l'heure.

P. D. : Il devait y avoir 5 Centres répartis sur le territoire national, il n'y en a, et selon toute vraisemblance, il n'y en aura, qu'un. Votre rapport au territoire régional est-il transformé par cette modification du projet global d'Archives Spécialisées « Monde du Travail»? -

G. M : La vocation est nationale, la direction nous ayant confié un rôle de collecte, de traitement et de communication des archives, mais aussi un rôle de coordination nationale sur ce type d'archives. Dans un nouveau contexte où faute d'un maillage du territoire, nous nous retrouvons seul centre spécialisé, comment concevons nous notre travail ? Effectivement, alors que nous nous étions assignés, au moment du démarrage, de la préfiguration du centre, un travail sur la Région Nord Pas de Calais, la Picardie, la Champagne, nous collectons maintenant des fonds d'Archives d'autres régions. On est amenés à collecter les archives de la société Bull - toute l'histoire de l'informatique depuis l'entre deux guerres -, on a travaillé avec la Compagnie Générale Maritime pour trouver des solutions pour ses fonds du Havre ; on travaille aussi avec la Compagnie Française d'Entreprises Métallurgiques, qui est « la» grosse entreprise de construction d'ouvrages d'arts, en France, mais aussi à l'étranger. Mais cela ne revient pas à tout rapatrier à Roubaix, y installer un monopole de l'archivage économique : ce serait ridicule. Dès lors, notre rôle consiste d'abord à recueillir les fonds d'entreprise que les Archives Nationales délocalisent pour donner à Roubaix une dimension suffisante pour être un centre ouvert à la recherche avec une taille critique suffisante. Nous collectons aussi les grandes entreprises à vocation nationale. En revanche, quand on est confronté à une entreprise d'une autre région, localisée dans sa production, et qui nous contacte pour essayer d'avoir un plan de travail pour sauvegarder ses archives, notre rôle national est d'essayer de trouver des solutions avec des partenaires locaux -archives départementales, municipales, travailler en coopération avec d'autres institutions non archivistiques aussi, de type associatif, d'économie mixte... On joue alors le rôle de structure de coordination, ayant son expérience comme structure spécialisée.

Le rôle national c'est aussi d'être un centre de ressources méthodologiques. C'est là quelque chose de très important, d'être un lieu où se crée une méthodologie du traitement de ces archives. La «formation» est un volet de notre activité directement lié à ce rôle.

10 P. D. : Vous collectez et communiquez des archives issues des acteurs des rapports sociaux de production, quelles spécificités cela induit-il dans votre manière de travailler?

G. M. : Qu'elles soient d'entreprise ou associatives, ce sont à plus de $90 \%$ des archives privées - je laisse de côté le problème juridique spécifique des grandes entreprises privées, puis nationalisées, puis privatisées... - Nous sommes donc un centre d'archives public, national, dépendant de l'Etat, mais qui ne collecte que des archives privées! Cela veut dire d'abord que nous n'avons pas à appliquer une réglementation comme on le ferait dans un cadre d'archives publiques. Ici, il n'y a pas d'obligation faite au producteur d'archives de verser régulièrement au Centre. Donc, nous ne pouvons travailler que de manière contractuelle sur une base conventionnelle, ...autour de contacts créés grâce aux études que nous pouvons faire sur des gisements d'archives existants, par les demandes des producteurs d'archives qui nous arrivent au fur et à mesure que nous sommes connus, par les contacts que nous avons par le biais d'intermédiaires, avec le rôle important des chercheurs dans ce domaine.

Cette base contractuelle impose des méthodes de travail précises. D’abord expliquer quel 
est notre rôle aux producteurs d'archives privées. En effet, il y a aussi, sur le marché, des entreprises d'archivage : elles proposent des prestations pour le traitement de l'information vivante dans l'entreprise, pour la conservation, par délégation de l'entreprise, des archives intermédiaires pendant le temps de vie juridique des documents. Nous, nous proposons des prestations patrimoniales, c'est-à-dire la conservation définitive de la mémoire pertinente de l'entreprise. Donc, là, il faut de la clarté lors du contact. L'organisation de l'information... on peut avoir notre mot à dire, ce n'est pas un sujet d'ignorance ou de désintérêt pour nous : il est très important de comprendre les structures actuelles de l'entreprise ne serait-ce que pour trouver, par méthode régressive, les structures de la mémoire et de la documentation. Mais, ce qui nous anime et qu'il nous faut partager, c'est un souci de la préservation du patrimoine en tant que mémoire d'une activité. A partir de là, on fait du traitement sur le site ; à partir du moment où les documents n'ont plus d'utilité active, on sélectionne ceux qui paraissent importants en tant que mémoire à pérenniser, on établit la convention avec le producteur d'archives.

Celle-ci traitera de la propriété (se déssaisit-il de sa propriété, ou -cas le plus fréquent- la conserve-t-il en nous confiant le dépôt ?) ; mais aussi de la communication. Puisque notre mission est de collecter des archives dans l'intérêt de la recherche historique, on a aussi le souci de les rendre communicables dans les meilleures conditions possibles, alors qu'une entreprise d'archivage qui a une mission de gestion ne se pose pas du tout ce problème.

On propose au producteur d'archives d'appliquer au fond les règles de la loi sur les archives publiques, et parfois même d'anticiper sur certains délais de communication établis par certaines de ces règles. Dans le monde archivistique, on est confronté en général au problème épineux du délais de 60 ans sur les archives dites sensibles... On a le délais de 30 ans, le plus souvent, et... on essaie de faire comprendre au producteur de ces archives que s'il a une démarche pour préserver sa mémoire, il faut qu'il ait aussi une démarche aussi pour l'ouvrir à la recherche. Mais on n'a pas une réglementation à appliquer, c'est au cas par cas, chaque convention de dépôt peut comporter une solution différente : tel peut demander l'habilitation préalable du chercheur, vouloir réserver la communication pendant tant d'années pour telle ou telle catégorie de documents, ou souhaiter la fermeture complète sur une période... Mais, là, on n'innove pas, c'est le principe appliqué pour les archives industrielles aux Archives Nationales. Comme archives publiques, notre particularité, c'est de ne travailler que sur une base conventionnelle.

P. D. : Jusqu'ici les usagers des Archives Publiques étaient essentiellement des historiens, spécalistes d'histoire économique et sociale, sans compter les généalogistes bien sûr. Avez-vous un public différent ? L'intérêt de nouveaux types de spécialistes, de chercheurs, pour le «Monde du Travail» et ses archives $a-t-i l$ des conséquences sur votre activité?

G. M. : Les archives du Monde de Travail (C.M.T), c'est à la fois un Centre d'Archives et un Centre culturel, avec des espaces d'exposition, des salles de conférences : les deux activités ne sont pas à nos yeux étanches ; elles peuvent amener ici des usagers intéressés différemment par la mémoire du monde du travail.

En ce qui concerne le public des chercheurs, la demande en documentation très, très contemporaine est beaucoup plus forte sur un centre d'archives du travail que sur d'autres archives. Parce que, au fond, il y a des mutations de société importantes sur la période et que le chercheur veut, à bon droit, les traiter à partir de documents produits 
directement par les acteurs du champ d'activité qu'il s'assigne. La création du C.M.T va beaucoup accélérer ce phénomène ; et ce n'est pas simple, notamment en ce qui concerne les problèmes de communicabilité. Dans notre domaine donc il y a une demande de documentation des années 70 , voire 80 , alors qu'à l'heure actuelle les grands débats des historiens du monde ouvrier et de l'histoire politique, sont centrés sur la seconde guerre mondiale, et bientôt la quatrième république. Je pense que nous allons devoir nous poser des problèmes d'ouverture de fonds très très récents. Et ça pose des problèmes méthodologiques - savoir traiter en temps réel - et des problèmes juridiques.

Mais l'ouverture à la recherche comporte aussi un autre aspect : alors que les archives sont traditionnellement le domaine de l'historien, histoire politique, sociale, économique...- ici, on s'aperçoit, pas à grande échelle certes, que viennent aussi d'autres chercheurs ; des juristes par exemple, qui s'intéressent au droit d'entreprise, mais pas un droit consistant uniquement en des textes réglementaires, non un droit lié à la jurisprudence produite par les rapports sociaux d'entreprise; viennent aussi des sociologues, des ethnologues (le patrimoine industriel est une source d'interrogations), des spécialistes des sciences humaines, comme vous, des sémiologues qui viennent chercher le support d'études sur le discours, sur l'écrit dans l'entreprise. C'est une ouverture intéressante. On s'aperçoit que notre spécialisation qui aurait pu donner l'impression que nous nous rétrécissions par rapport à un centre d'archives générales, donne en fait des potentialités de débouchés sur l'ensemble des sciences humaines.

C'est intéressant et stimulant ; et en même temps, ça nous amène aussi à réfléchir sur les propres méthodes que nous nous donnons pour la collecte... et surtout pour l'échantillonnage des archives.

En effet, la réalité des archives contemporaines, c'est que la masse est telle que pratiquement, on est amené à conserver un ratio de $10 \%$ de la production. C'est à peu près comme ça dans tous les pays industriels. Alors, dans les archives publiques, on s'est créé des méthodes d'échantillonnage, en conjuguant l'expérience des archivistes et les contacts avec les universitaires ; cela a donné une base de travail. On s'aperçoit que l'ouverture à d'autres types de recherche nous amène à nous interroger sur la pertinence de certaines éliminations que nous faisions. Je pense aux parapheurs, aux séries de télex qui sont la circulation interne de l'entreprise. C'est vrai qu'on avait tendance à considérer que c'est un support fragile, et sans contenu informatif très fort... Bon, il est évident qu'il $\mathrm{y}$ a de nouvelles entrées, de nouveaux problèmes, ça nous amène à nous dire qu'il ne faut pas qu'on se " plante ». Car on travaille quand même pour le long terme. On ne peut travailler efficacement que si on a un lien avec les chercheurs, un lien quasi institutionnel. Cela reste à créer, mais il est nécessaire si nous voulons avoir une réflexion en amont sur les phases de collecte et de traitement.

\section{Entretien avec Armelle Desplanque-Le Goff, conservateur du patrimoine, Archives du monde du travail}

Nous avons demandé à Armelle Desplanque de donner aux lecteurs d'Etudes de Communication une idée de ce pouvaient représenter des " fonds d'archives d'entreprises »...

Pour présenter ces fonds, il faut à la fois avoir une idée des masses volumineuses de documents ou des épaves que nous devons expertiser dans des conditions matérielles 
souvent difficiles (caves, greniers, etc.). Il faut encore avoir une idée des procédures et des instruments intellectuels que nous avons à notre disposition pour définir des modalités de tri et d'éliminations. En effet la première chose, c'est de maitriser et d'organiser cette masse d'archives ; il est pour nous hors de question d'investir des moyens financiers et humains importants pour collecter des masses volumineuses mais pauvres en informations ou des fonds trop fragmentaires pour être utilisables.

Il nous faut donc mettre au point une méthodologie de traitement et concevoir des inventaires qui offrent des points d'ancrage pour le chercheur. Une étude sur la typologie des documents nous a paru prioritaire pour préparer les entrées, définir les méthodes de travail et produire des instruments de recherche utilisables par tout public.

\section{Des questions pour anticiper sur la nature de l'entreprise et de sa production d'archives.}

Chaque fonds d'entreprise est une réalité unique. Notre première démarche, avant d'en entreprendre la collecte et le classement est donc de tenter de répondre à quelques questions claires et précises : quelle est la forme juridique de l'entreprise concernée ? Quel est son secteur d'activité? Quelle est sa finalité? Est-ce la production de biens destinés à la vente et ou le négoce de ces biens ? Est-ce la fourniture de services ? Quelle est la spécificité de ses activités? Quel est son type de fabrication (non standardisé, c'està-dire des fabrications spécifiées par le client sur commande ou par l'entreprise sur devis, ou, au contraire, standardisé, c'est-à-dire des fabrications sur catalogues)? Quel est son rythme de fabrication? Que savons-nous, en outre, de sa dimension, de son implantation et de sa concentration géographique, de son marché, de son degré de concentration ou d'intégration verticale ou horizontale ? Les documents reçus et produits par l'entreprise dépendent en effet très largement et très concrètement de toutes ces caractéristiques. Ces informations recueillies au préalable facilitent le travail d'appréhension des masses et de compréhension des documents.

\section{Une typologie prenant en compte la nature juridique des documents archivés.}

17 En partant du critère juridique, il est possible de déterminer une première typologie des documents. Toutes les sociétés commerciales sont dotées, sur le plan juridique, de la personnalité morale. En conséquence elles sont soumises à de nombreuses obligations imposées par le droit des sociétés, le code du commerce, le code des impôts, le code du travail et de la sécurité sociale. De par l'intervention du législateur, des règles strictes pèsent sur la production de documents sociaux, de documents comptables et de documents relatifs au personnel qui peuvent être semblables d'une société à l'autre quant à leur aspect diplomatique.

C'est déjà là donc une masse de documents " obligatoires » : actes de société ; documents émanant de conseils et d'assemblées; documents comptables (livre-journal, grand livre, inventaire...) ; documents patrimoniaux (titres de propriété, plans et dossiers techniques de bâtiments et de machines, brevets d'invention, dossiers de marques de fabrique et de dessins et modèles...) ; documents relatifs au personnel (registre du mouvement, registre d'inscription des employés et ouvriers âgés de moins de 18 ans, livres de paie, registre des travailleurs étrangers, registre des accident du travail, registre des mises en demeure de 
l'Inspection du Travail...) et encore, avec le développement de la voie collective pour le règlement des rapports de travail, dossiers d'élections et registres de délibérations du Comité d'Entreprise.

Tous ces documents, outre leur intérêt propre, traduisent le climat social, témoignent du décalage entre le droit et la pratique au quotidien, des écarts entre les tendances globales et l'évolution spécifique à certaines branches d'activité ou à certaines régions géographiques.

Sur la durée, des évolutions se font sentir, qui peuvent être intéressantes à noter. Les registres de Conseil d'administration par exemple, très détaillés à la fin du 19ème siècle, et qui finissent par simplement résumer des décisions. Il est aussi intéressant de trouver de nos jours, de manière peu régulière il est vrai, les archives de comités d'études ou de direction. On peut, dans ces comptes-rendus remarquer comment avant guerre certains comités de direction s'apparentaient plus à des conseils de famille, avec une structure familiale classique dans les entreprises du Nord, où l'on trouvait le Service de Mr Jacques, le Service de Mr Eugène...

\section{Pour organiser la masse documentaire, penser les fonctions dans l'entreprise...}

Mais le cadre juridique ne permet pas de prendre en compte une masse importante de documents qui ne relèvent pas d'une obligation juridique. Il existe des " archives stratégiques ", des " archives techniques» qui permettront de saisir précisément toutes ces pratiques d'écriture qui vous intéressent. Une autre perspective typologique est alors nécessaire. Tous les types de documents, obligatoires ou pas, quel que soit leur support, résultent d'une activité ; il est donc indispensable pour les comprendre d'avoir quelques notions sur l'organisation de l'entreprise. La définition des différentes fonctions de l'entreprise permet, alors, d'élaborer une méthode de travail pour faciliter la compréhension des fonds. La fonction constitue le pont entre le projet de l'entreprise, c'est-à-dire sa politique et ses objectifs, et les moyens qui lui sont nécessaires pour réaliser ce projet.

Une première description des entreprises par le biais de leurs fonctions a été réalisée dans l'ouvrage d'Henri Fayol, Administration industrielle et générale (1916). Sa définition de l'entreprise se résume en cinq impératifs (prévoir, organiser, commander, coordonner et contrôler). Il distinguait ensuite six fonctions dans une entreprise :

la fonction technique : produire, fabriquer, transformer

la fonction commerciale : acheter, vendre, échanger

la fonction financière : rechercher et gérer des capitaux

la fonction sécurité : protéger les biens et les personnes

la fonction comptabilité : établir les inventaires, les bilans, les coûts de revient et les statistiques

la fonction administrative qui accompagne les autres fonctions.

Cette distinction reflète la mentalité et les préoccupations de l'époque : Fayol ne prévoit pas de fonction « personnel » ou " gestion des ressources humaines ». Actuellement, nous suivons le modèle proposé par Jean Gerbier et nous distinguons la fonction vente ; la fonction technique et de production ; la fonction approvisionnement; la fonction personnel ; les fonctions administratives ; la fonction finance et comptabilité ; la fonction direction. La fonction est conçue comme une réalité abstraite, non directement 
observable, et se distingue de « l'organe », directement observable.

Cette analyse des documents sous cet éclairage nous permet de résoudre les énigmes de certains organigrammes. Ainsi les «secrétaires généraux », d'une entreprise à une autre n'ont pas le même rôle. Dans certaines entreprises, il est important d'identifier l'éminence grise, le bras droit du directeur ou la personnalité forte de la famille. Dans telle grande entreprise textile, toutes les notes passaient par « Mr Marcel ». Il contrôlait tout ; même dans les dossiers techniques, on reconnaît sa griffe. On reconnaît aussi ce rôle directorial quand c'est son bras droit qui signe.

\section{Un observatoire des rapports sociaux}

19 On peut voir aussi, à la lecture de ces archives, l'évolution des mentalités dans les rapports sociaux, notamment avec le personnel. Dans le textile, la construction mécanique, quand on compare sur une longue période, on peut voir le vocabulaire changer en même temps que la façon de considérer le personnel. Dans les années 1920, c'est le comptable qui tient à jour le registre du personnel, et puis, petit à petit, ça va devenir le service des relations industrielles, des relations sociales, des relations humaines, et maintenant des ressources humaines. C'est tout un vocabulaire qui change, mais aussi les objectifs : on peut voir émerger les documents de formation, le journal d'entreprise, les livrets d'accueil, le ancêtres des cercles de qualité, dans les années 1955, avec les commissions qui visaient à améliorer le rendement. La période d'après guerre, avec l'établissement des C.E. voit se développer le souci de la formation professionnelle, de la sécurité, avec la médecine du travail...

On peut voir aussi, à la lecture des archives l'autre face des rapports sociaux, la méfiance, notamment dans la période des grandes grèves: les services "personnel », ou «relations humaines » collectent tous les tracts, même ceux distribués à la porte des usines, avec des annotations portées sur les conditions dans lesquelles ils ont été distribués. On voit aussi les rapports sur tout ce qui se faisait, sur les élections...

\section{Des archives de la production propre de documents, mais aussi de « l'information » recueillie}

Il y a en effet un aspect des archives qui est le résultat de l'information qui circule dans l'entreprise. On peut ainsi repérer "l'information de source intérieure ", qui va se traduire par des circulaires, des notes de service, des comptes-rendus de réunions. Et il y a « l'information de source extérieure » venant d'autres organismes professionnels, des syndicats patronaux, de l'Etat. Des coupures de presse sont recueillies, par le service de relations publiques ou par le secrétariat général. Ici encore. on note une évolution, surtout dans les années 1970 : un souci de plus en plus grand de l'image de l'entreprise. Comment l'entreprise est perçue? La question ne se règle plus seulement par les cercles de qualité, à l'interne, par une attention aux rapports sociaux dans l'entreprise. On cherche à savoir, et on collecte pour cela des informations, comment l'entreprise est perçue à l'extérieur. Communiqués de presse, et, dans les grandes entreprises, actions de mécénat. Mais encore action publique en cas de crise, de catastrophe. On trouve ici des informations sur de nombreux supports, $\mathrm{y}$ compris les cassettes vidéos où sont enregistrés les grands moments de la vie de l'entreprise, vus par le regard des médias ou par ses propres productions. 


\section{Les relations avec la clientèle}

21 avons un fonds d'imprimerie qui comporte la correspondance avec la clientèle sur une très très longue période. Le cas est intéressant car dans cette entreprise, tout était classé par ordre alphabétique de client, dans des casiers, et à la fin de l'année, c'était relié : on gardait tout, aussi bien les bordereaux d'envoi... C'est une source très riche de pratiques de 1945 à 1990. On peut y voir l'évolution géographique de la clientèle, mais on peut aussi bien étudier l'évolution des pratiques d'écriture et de leurs aspects diplomatiques. Un aspect sociologique peut aussi être envisagé : les fiches clients peuvent avoir un aspect inquisiteur, il s'agit de savoir si les clients sont solvables...

\section{Pour une sémiologie des écrits d'entreprise}

L'imprimerie est aussi stratégique pour repérer l'évolution même des produits imprimés des entreprises. On peut lire l'évolution des demandes, l'évolution des techniques de production.

Dans cette imprimerie qui avait une très large clientèle sur la France, on faisait aussi bien avant guerre de la typographie d'entreprise, imprimant des bulletins de paie, des notices d'information... une très belle collection d'imprimés d'entreprise. Mais, en outre, sa grande spécialité, c'était les impressions couleur : étiquettes pour l'emballage et le cartonnage. On a pu ainsi retrouver des premières étiquettes de clients fort connus, des dossiers de fabrication, de 1923 à 1990. Des dossiers administratifs suivaient le produit de la commande jusqu'à l'envoi, avec des papiers sur la quantité d'encre pour réaliser telle étiquette, etc... Contrôle de production, facturation. Mais ce qui est intéressant, c'est qu'on a pu conserver les épreuves et un exemplaire de chaque produit fini. On est en train de faire un fichier par client et par produit, et il y en a des milliers et des milliers. Nous avons aussi la correspondance, avec les demandes des clients, en particulier des cas où le travail demandé fait référence au « modèle » d'une autre marque de renom. Bien sûr, un tel gisement peut intéresser des maquettistes, mais aussi des sémiologues, des chercheurs en communication.

Entretiens réalisés par P. Delcambre, Gérico/Lille 3, février 1995.

\section{BIBLIOGRAPHIE}

Ouvrages généraux sur les archives d'entreprises.

Gille, B., (1957), Etat sommaire des archives d'entreprises conservées aux Archives Nationales, Ed. Archives Nationales. 40 p.

Guerin-Brot, I., (1989), Les Archives des entreprises : conseils pratiques d'organisation, 2ème ed, Archives nationales, 87p. 
Hamon, M., (1987), « Archives d'entreprises, histoire industrielle et histoire sociale : évolutions et perspectives ", Bulletin du Comité des archives d'entreprises du Conseil International des archives, $\mathrm{n}^{\circ}$ 10,1987 , p. 5-10.

Sur l'organisation de l'entreprise

Gerbier, J., (1993), Organisation et fonctionnement de l'entreprise, Lavoisier. 